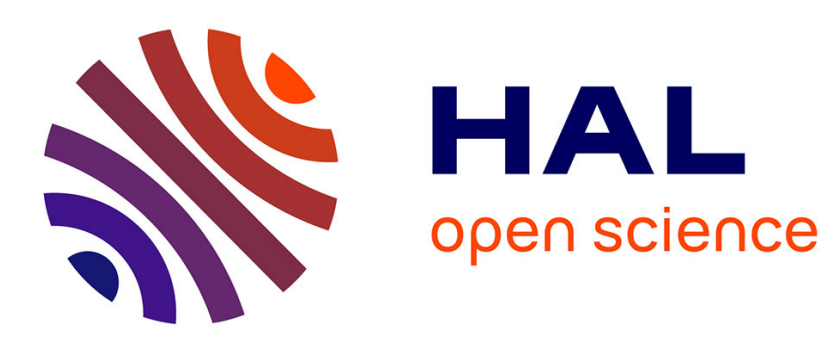

\title{
Spectral efficiency of energy efficient multicarrier systems
}

\author{
Majed Haddad, Oussama Habachi, Piotr Wiecek, Yezekael Hayel
}

\section{To cite this version:}

Majed Haddad, Oussama Habachi, Piotr Wiecek, Yezekael Hayel. Spectral efficiency of energy efficient multicarrier systems. ACM SIGMETRICS Performance Evaluation Review, 2014, 42 (2), pp.24-26. 10.1145/2667522.2667529 . hal-01101858

\section{HAL Id: hal-01101858 \\ https://inria.hal.science/hal-01101858}

Submitted on 9 Jan 2015

HAL is a multi-disciplinary open access archive for the deposit and dissemination of scientific research documents, whether they are published or not. The documents may come from teaching and research institutions in France or abroad, or from public or private research centers.
L'archive ouverte pluridisciplinaire HAL, est destinée au dépôt et à la diffusion de documents scientifiques de niveau recherche, publiés ou non, émanant des établissements d'enseignement et de recherche français ou étrangers, des laboratoires publics ou privés. 


\section{Spectral Efficiency of Energy Efficient Multi-carrier Systems}

\author{
Majed Haddad \\ INRIA Sophia-Antipolis, \\ France
}

\author{
Oussama Habachi \\ University of Avignon, France \\ Yezekael Hayel \\ University of Avignon, France
}

\author{
Piotr Wiecek \\ Wroclaw University of \\ Technology, Poland
}

\begin{abstract}
We investigate the achievable performances of multi-carrier energy efficient power control game. Both the simultaneousmove and the hierarchical games are addressed. For the first time, we derive analytical closed-form expressions of the spectrum coordination and the spectral efficiency of such models. Our results indicate that the spectrum coordination capability induced by the power control game model enables the wireless network to enjoy the energy efficiency improvement while still achieving a high spectral efficiency. Such an important result offers insights into how to design power control in multi-carrier radio environments.
\end{abstract}

\section{INTRODUCTION}

In order to cope with the exponential increase in their newly deployed networks, operators are compelled to find new ways to boost their network capacity, provide better coverage, and ease network congestion. However, achieving these goals is usually at the expense of higher energy consumption. In recent years, energy and power efficient designs of communication and computing networks have become more crucial because of the steadily rising energy cost and environmental concerns [1]. There is thus an urgent need to address the energy efficiency in such networks along with wide variety of delay and throughput objectives. Furthermore, multi-carrier transmission are steadily attracting more attention in wireless communications after its adoption in several recent wireless communications systems standards such as LTE.

We shall consider a power control game in multi-carrier energy efficient wireless networks. Each user can choose freely his transmit power over the carriers in order to selfishly maximize a certain individual performance criterion, called utility (or payoff) in the context of game theoretic studies. Unlike many works concerning this problem, the chosen users' utility is not the transmission rate, e.g., [2] but the energy efficiency of their communications.

In a prior work [3], we proposed a hierarchical game theoretic model for two-user-two-carrier energy efficient wireless systems. It was shown that, under some assumptions, users choose their transmitting carriers in such a way that if the leader transmits on a given carrier, the follower have incentive to choose the other carrier. In this work, we extend the original problem in [3] to some general models that can

IFIP WG 7.3 Performance 2014, October 7-9, Turin, Italy. Copyright is held by author/owner(s). be widely used in practice assuming an arbitrary number of carriers. We derive analytical closed-form expressions of the spectrum coordination and the spectral efficiency of such models considering i.i.d. and correlated fading channel. $\mathrm{Nu}-$ merical results illustrate the balance between the achievable rate and energy consumption of the system.

\section{SYSTEM MODEL}

We consider a decentralized multiple access channel with an arbitrary number of carriers $K \geq 2$ composed of a leader (the user with the higher priority - indexed by 1 ), having the priority to access the medium, and a follower (the user with the lower priority)- indexed by 2) that accesses the medium after observing the action of the leader. Without the constraint of exclusive assignment of each carrier for users, we shall consider the interference from other user's signals on the same carrier. For any user $n \in\{1,2\}$, the received signal-to-noise plus interference ratio (SINR) is expressed as

$$
\gamma_{n}^{k}=\frac{g_{n}^{k} p_{n}^{k}}{\sigma^{2}+\sum_{\substack{m=1 \\ m \neq n}}^{2} g_{m}^{k} p_{m}^{k}}
$$

where $g_{n}^{k}$ and $p_{n}^{k}$ are resp. the fading channel gain and the power control of user $n$ transmitting on carrier $k$, whereas $\sigma^{2}$ stands for the variance of the Gaussian noise. We statistically model the channel gains $g_{n}^{k}$ to be independent identically distributed (i.i.d.) over the Rayleigh fading coefficients.

\section{NETWORK ENERGY EFFICIENCY ANAL- YSIS}

The energy efficiency system model adopted throughout the paper is based on the seminal paper [4] that defines the energy efficiency framework. The energy efficiency can be concisely captured by an increasing, continuous and Sshaped "efficiency" function $f(\cdot)$, which measures the packet success rate, where $R_{n}$ is the transmission rate of user $n$ over carrier $k$. The following utility function allows one to measure the corresponding trade-off between the transmission benefit (total throughput over the $K$ carriers) and cost (total power over the $K$ carriers):

$$
u_{n}\left(\mathbf{p}_{\mathbf{1}}, \mathbf{p}_{\mathbf{2}}\right)=\frac{R_{n} \cdot \sum_{k=1}^{K} f\left(\gamma_{n}^{k}\right)}{\sum_{k=1}^{K} p_{n}^{k}}
$$


where $\mathbf{p}_{\mathbf{n}}$ is the power allocation vector of user $n$ over all carriers, i.e., $\mathbf{p}_{\mathbf{n}}=\left(p_{n}^{1}, \ldots, p_{n}^{K}\right)$. The utility function $u_{n}$, that has bits per joule as units, perfectly captures the tradeoff between throughput and battery life and is particularly suitable for applications where energy efficiency is crucial.

\section{THE GAME THEORETIC FORMULA- TION}

\subsection{The simultaneous-move game problem}

An important solution concept of the game under consideration is the Nash equilibrium (NE), which is a fundamental concept in non-cooperative strategic games. It is a vector of strategies (referred to hereafter and interchangeably as actions) $\mathbf{p}^{N E}=\left\{\mathbf{p}_{\mathbf{1}}{ }^{N E}, \mathbf{p}_{\mathbf{2}}{ }^{N E}\right\}$, one for each player, such that no player has incentive to unilaterally change his strategy, i.e., $u_{n}\left(\mathbf{p}_{\mathbf{n}}{ }^{N E}, \mathbf{p}_{-n}^{N E}\right) \geq u_{n}\left(\mathbf{p}_{\mathbf{n}}, \mathbf{p}_{-n}^{N E}\right)$ for all action $\mathbf{p}_{\mathbf{n}} \neq \mathbf{p}_{\mathbf{n}}{ }^{N E}$, where the $-n$ subscript on vector $\mathbf{p}$ stands for "except user $n$ ".

\subsection{The hierarchical game formulation}

Hierarchical models in wireless networks are motivated by the idea that the utility of the leader obtained at the Stackelberg equilibrium can often be improved over his utility obtained at the NE when the two users play simultaneously. In a Stackelberg game framework, a foresighted follower adapts his power allocation vector $\mathbf{p}_{\mathbf{2}}$, based on the power vector of the leader $\mathbf{p}_{\mathbf{1}}$ in advance. The power allocation of the shortsighted leader will re-embody in the form of interference introduced to the foresighted follower as given by Eq. (2).

DEFINITION 1. (Stackelberg equilibrium): A vector of actions $\widetilde{\mathbf{p}}=\left(\widetilde{\mathbf{p}}_{\mathbf{1}}, \widetilde{\mathbf{p}}_{\mathbf{2}}\right)=\left(\widetilde{p}_{1}^{1}, \ldots, \widetilde{p}_{1}^{K}, \widetilde{p}_{2}^{1}, \ldots, \widetilde{p}_{2}^{K}\right)$ is called Stackelberg equilibrium (SE) if and only if:

$$
\widetilde{\mathbf{p}}_{\mathbf{1}}=\arg \max _{\mathbf{p}_{\mathbf{1}}} u_{1}\left(\mathbf{p}_{\mathbf{1}}, \bar{p}_{2}\left(\mathbf{p}_{\mathbf{1}}\right)\right),
$$

where for all $\mathbf{p}_{\mathbf{1}}$, we have

$$
\bar{p}_{2}\left(\mathbf{p}_{\mathbf{1}}\right)=\arg \max _{\mathbf{p}_{\mathbf{2}}} u_{2}\left(\mathbf{p}_{\mathbf{1}}, \mathbf{p}_{\mathbf{2}}\right),
$$

and $\widetilde{\mathbf{p}}_{\mathbf{2}}=\bar{p}_{2}\left(\widetilde{\mathbf{p}}_{\mathbf{1}}\right)$.

In the remainder, we shall consider to compute closed-form expressions of the achievable performance for the simultaneousmove game and the hierarchical game.

\section{SPECTRUM COORDINATION}

In this section, we shall look for values of power gains of users that may lead the leader and the follower to use the same carrier. More specifically, the next proposition shows that the probability of no coordination between the players is always small and decreases fast as the number of carriers grows.

Proposition 1. The probability that there is no coordination between the players in the hierarchical model is bounded above by

$$
\left(1+\gamma^{*}\right) \mathcal{B}\left(1+\gamma^{*}, K\right)\left[\frac{K-1}{K}+\left(1+\gamma^{*}\right) \mathcal{B}\left(1+\gamma^{*}, K\right)\right]
$$

which is the exact probability of no coordination in the Nash version of the model. $\mathcal{B}$ denotes the Beta function and $\gamma^{*}$ is the unique (positive) solution of the first order equation

$$
x f^{\prime}(x)=f(x)
$$

which has a unique solution if the efficiency function $f(\cdot)$ is sigmoidal. Moreover, it is easy to see that the probability derived above is $O\left(K^{-\left(1+\gamma^{*}\right)}\right)$.

REMARK 1. In the above proposition, we assume that the channel gains for different carriers of each of the users are i.i.d. Rayleigh random variables, which is typically the case when the two carriers are far enough [5]. Otherwise, the probability computed there can be treated as an upper bound for respective probabilities, when there is a positive correlation between different carriers of each of the users, which is much more realistic. We will see later in the paper (see Fig. 1) that, in the case of positive correlation over carriers, these probabilities will be even smaller (and so faster decreasing to 0 ).

\section{SPECTRAL EFFICIENCY}

Along with energy efficiency, spectral efficiency - defined as the throughput per unit of bandwidth - is one of the key performance evaluation criteria for wireless network design. These two conflicting criteria can be linked through their trade-off [6]. In fact, the optimal energy efficiency performance often leads to low spectral efficiency performance and vice versa [7]. Therefore, it is often imperative to make a trade-off between energy efficiency and spectral efficiency. In the following, we give a closed-form expression of the lower bound on the sum spectral efficiency of the Stackelberg model.

Proposition 2. The spectral efficiency in the hierarchical model in case there is a coordination between the users is strictly bigger than

$$
\left[1-\left(1+\gamma^{*}\right) \mathcal{B}\left(1+\gamma^{*}, K\right)\left(\frac{K-1}{K}+\left(1+\gamma^{*}\right) \mathcal{B}\left(1+\gamma^{*}, K\right)\right)\right] \cdot \log _{2}\left(1+\gamma^{*}\right)
$$

which is equal to the spectral efficiency in the simultaneousmove game.

The computation done in Proposition 2 is done only in case there is coordination between the players. This means that this is only a lower bound for the total spectral efficiency in our model. However, by Proposition 1, it becomes very tight as $K$ goes to infinity. An easy consequence of this is that the spectral efficiency in the limit model (with an infinite number of carriers) can be computed exactly, and is equal to $\log _{2}\left(1+\gamma^{*}\right)$.

\section{SIMULATIONS}

We consider the well-known energy efficiency function in power allocation games, $f(x)=\left(1-e^{-x}\right)^{M}$, where $M=100$ is the block length in bits. For this efficiency function, $\gamma^{*} \simeq 6.4$ (or $\left.8.1 \mathrm{~dB}\right)$. Simulations were carried out using a rate $R_{n}=1 \mathrm{Mbps}$ for $n=\{1,2\}$. The fading channel is modeled as a quasi-static correlated Rayleigh-fading following the model in [8].

Figure 1 illustrates the effect of the correlation between different carriers of each of the users on the probability of no coordination for the Stackelberg and the Nash game. For i.i.d. Rayleigh fading, it is clearly shown that the theoretical closed-form expression derived in Eq. 3 matches the simulated probability of no coordination in the Nash case 


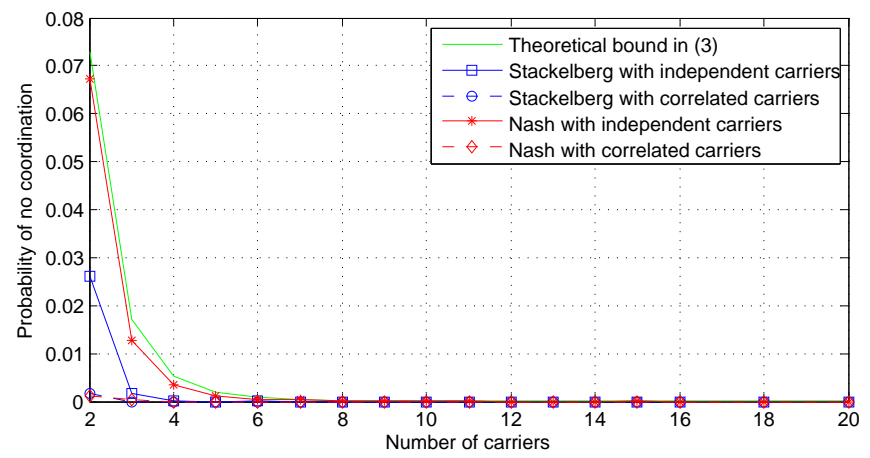

Figure 1: The probability of no coordination between the players.

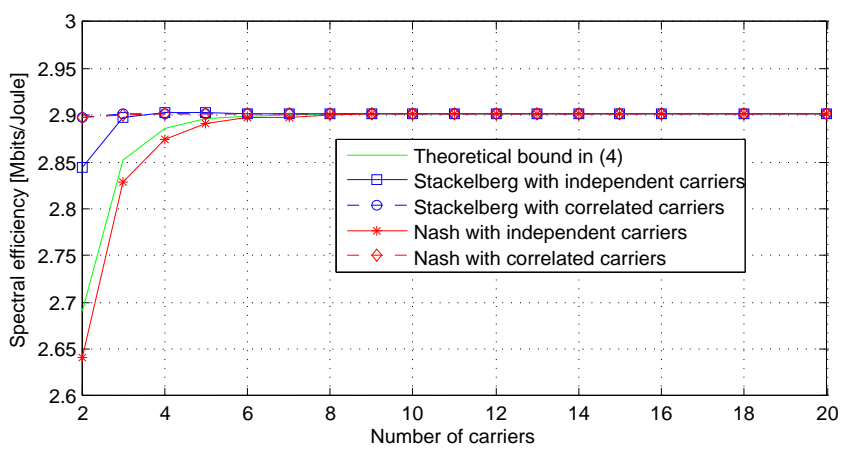

Figure 2: Average spectral efficiency.

while it upper bounds the probability of no coordination in the the Stackelberg game which demonstrates good accuracy of the results derived within the paper. In the case of correlated fading over carriers, as we expected in Section 5, results in Fig. 1 show that the simulated probability of no coordination is even smaller than for i.i.d. fading channels and so faster decreasing to 0 even for a moderate number of carriers $K$.

In Fig. 2, we compare the closed-form bound on the spectral efficiency derived in Eq. 4 with the simulated spectral efficiency at the Nash and Stackelberg equilibria. We first notice that, for i.i.d. fading channel, the Stackelberg model outperforms the Nash model in terms of average spectral efficiency. Moreover, comparing the simulated and the theoretical spectral efficiency in (4), we observe that the closedform bound turns out to be very tight. Of particular interest is the fact that, for correlated fading channels, both the Stackelberg and the Nash models achieve better than in the case of i.i.d. fading channels.

In order to illustrate the balance between the achievable rate and energy consumption of the system, we plot in Fig. 3 the spectral efficiency as a function of the energy efficiency. It is clearly shown that the proposed Stackelberg decision approach maximizes the energy efficiency while still optimizing the spectral efficiency which is in contrast with most related works so far in which the optimal energy efficiency performance often leads to low spectral efficiency performance and vice versa [7]. All these key features have a great impact on the network performance, and may further guide the design of power control strategies in a multi-carrier

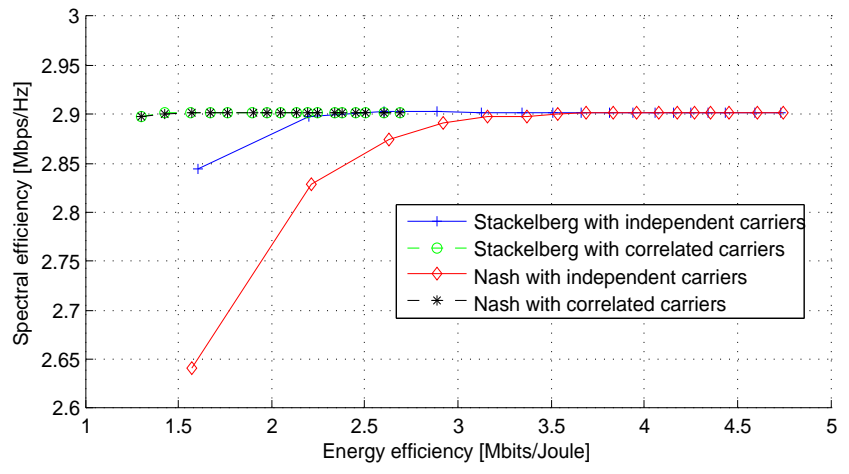

Figure 3: Spectral efficiency vs. Energy efficiency.

environments.

\section{CONCLUSION}

In this work, we have studied the achievable performance of multi-carrier energy efficient power control game. For the first time, our analysis has given closed-form expressions of the spectrum coordination and spectral efficiency. It has been shown that the throughput scaling of such schemes is $\log _{2}\left(1+\gamma^{*}\right)\left(1-O\left(K^{-\left(1+\gamma^{*}\right)}\right)\right)$, while a vanishing fraction of the carriers may suffer from mutual interference as the number of the carriers goes large. Simulation results shows that correlation over carriers is a suitable feature as it brings more coordination - and thus leads to a better spectral efficiency - desirable for the design of future wireless networks. This suggests that the network planning and design phase should rather exploit carrier correlation by allocating very close carriers to users as channel characteristics are likely to be similar.

\section{Acknowledgment}

This work has been partially supported by the European Commission within the framework of the CONGAS project FP7-ICT-2011-8-317672, see www.congas-project.eu.

\section{REFERENCES}

[1] C. Han, T. Harrold, S. Armour, I. Krikidis, S. Videv, P. M. Grant, H. Haas, J. Thompson, I. Ku, C.-X. Wang, T. A. Le, M. Nakhai, J. Zhang, and L. Hanzo, "Green radio: radio techniques to enable energy-efficient wireless networks," IEEE Communications Magazine, vol. 49, no. 6, pp. 46-54, 2011.

[2] L. Lai and H. E. Gamal, "The Water-Filling Game in Fading Multiple-Access Channels," IEEE Transactions on Information Theory, vol. 54, no. 5, pp. 2110-2122, 2008.

[3] M. Haddad, Y. Hayel, and O. Habachi, "Spectrum coordination in energy efficient cognitive radio networks," IEEE Transactions on Vehicular Technology, 2014.

[4] D. Goodman and N. Mandayam, "Power control for wireless data," IEEE Personal Communications, vol. 7, pp. 48-54, 2000.

[5] J. G. Proakis and M. Salehi, Communication Systems Engineering, 2nd ed. Upper Saddle River, NJ, USA: Prentice-Hall, August 2001.

[6] F. Heliot, M. Imran, and R. Tafazolli, "On the energy efficiency-spectral efficiency trade-off over the mimo rayleigh fading channel," Communications, IEEE Transactions on, vol. 60, no. 5, pp. 1345-1356, 2012.

[7] Y. Chen, S. Zhang, S. Xu, and G. Y. Li, "Fundamental tradeoffs on green wireless networks," Communications Magazine, IEEE, vol. 49 , no. 6 , pp. 30-37, Jun. 2011.

[8] K. Baddour and N. Beaulieu, "Autoregressive modeling for fading channel simulation," Wireless Communications, IEEE Transactions on, vol. 4, no. 4, pp. 1650-1662, July 2005. 